\title{
Pathological Analysis of Diffuse Large B-Cell Lymphoma and Clinical Treatment with Chinese and Western Medicine
}

\author{
Xianhui Liü, 2\#, a , Xiaona Guo ${ }^{3 \#, b}$ and Chunsheng Yan ${ }^{3, c}$ \\ ${ }^{1}$ Henan Province Hospital of TCM, Zhengzhou, 450000, Henan, P. R. China \\ ${ }^{2}$ The Second Affiliated Hospital of Henan University of Traditional Chinese Medicine, Zhengzhou, \\ 450000, Henan, P. R. China \\ ${ }^{3}$ Huanghe Science and Technology College, Zhengzhou, Henan, P. R. China

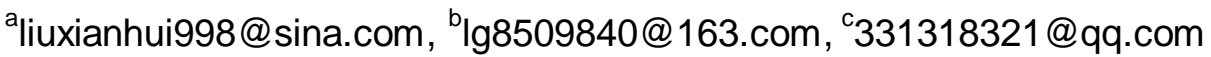 \\ *The corresponding author
}

\#Liu Xianhui and Guo Xiaona contributed equally to this work and should be considered co-first

Keywords: Diffuse large B cell lymphoma; Pathogenesis; Clinical manifestations; Clinical treatment

\begin{abstract}
By exploring the pathogenesis and clinical manifestations of diffuse large B-cell lymphoma, combining Chinese and Western medicine and other treatment for post treatment of diffuse large B-cell lymphoma provides clinical experience.
\end{abstract}

\section{Introduction}

Diffuse large B-cell lymphoma (DLBCL) is the most common adult lymphoma type, and is a group of malignant tumors and many great heterogeneity in clinical presentation, morphology and prognosis. In western countries, the incidence of DLBCL accounts for non-Hodgkin's lymphoma (NHL) of 31\%, in Asian countries accounted for more than $40 \%$ of the NHL, while China's incidence of the lack of precise statistics. DLBCL can occur at any age, but more common in the elderly, age of onset is 60-64 years old, men slightly more than women. Clinically, painless mass increases rapidly as a typical example, about one-third of patients with B symptoms, the incidence of bone marrow involvement was $16 \%$ [1]. Cancer occurs mainly in the lymph nodes, the patient in excess of about $30 \%$ of the performance limitations of extranodal starting lesions. Extranodal lesions common in the gastrointestinal tract, bone and central nervous system. DLBCL tumor showed diffuse large B-cell growth, tumor cell nuclei the normal tissue cells of the same size or larger than the nuclei of the cells nucleus, typically greater than 2 times normal lymphocytes. As shown in Fig. 1.

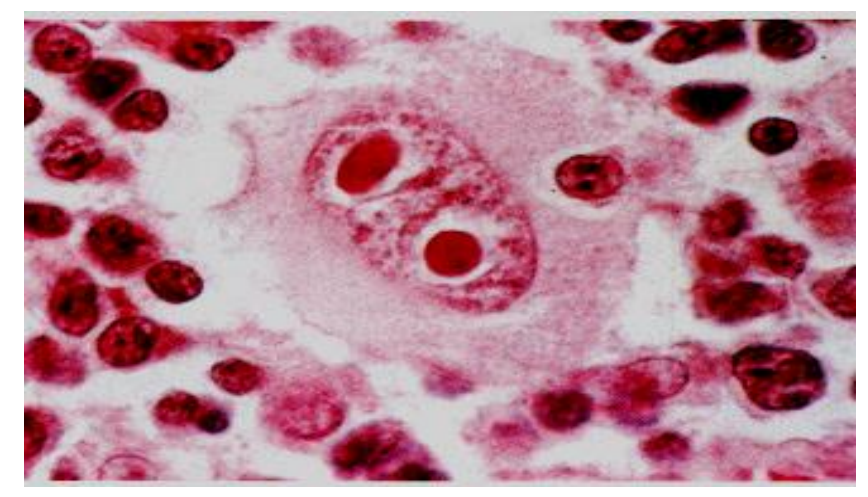

Figure 1. Hodgkin's lymphoma cell mirror image (taken from Rubin Pathology 3rd 1998)

According to morphology, WHO classification DLBCL may include a central mother cell type, immunoblastic type, rich in T cell / tissue type, anaplastic type, plasma cell type and female full-length ALK expression. Because these subtypes of clinical treatment and prognosis of no clear meaning, WHO classification will be classified as a class. Typical immunohisto chemical expression DLBCL is: 
$\mathrm{CD} 20$ +, CD45 +, CD79a +, Ki67 +, CD3-. Molecular genetic testing can show bcl-2, bcl-1, c-myc antigen receptor gene rearrangement [2].

DLBCL is a multi-gene effects caused by tumors, show heterogeneity genetics. With the advent of DNA microarray technology, according to different patterns of gene expression, DLBCL into the germinal center B cell-like lymphoma, activated B cell-like lymphoma, and a third type DLBCL (type 3 DLBCL). DNA microarray technology to determine the molecular expression profiling to predict survival of DLBCL, as shown in Fig. 2.

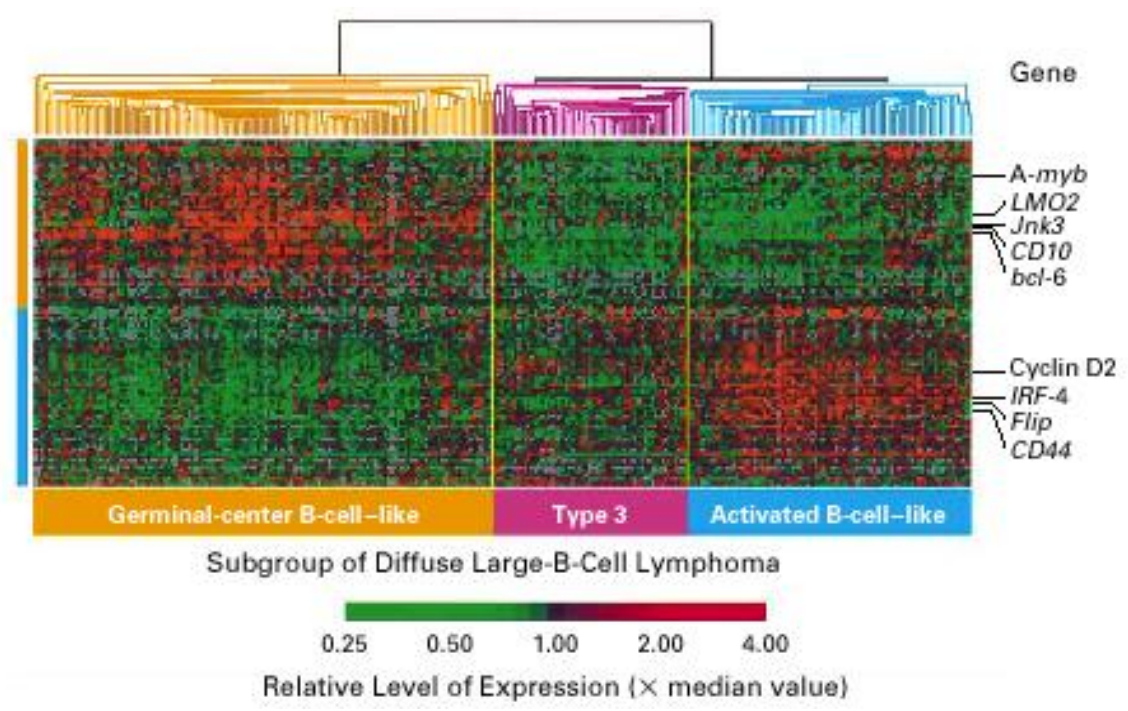

Figure 2. Subgroup of diffuse large-b-cell lymphoma

\section{Diffuse Large B-Cell Lymphoma Analysis}

Clinical Manifestations. Clinical manifestations, patients usually rapidly enlarged lymph nodes $10 \%$ $-15 \%$ of patients with bone marrow involvement $40 \%-50 \%$ of patients had extranodal disease patients often develop fever, night sweats, progressive weight loss and other symptoms. The rapid progression of the disease, which if not actively treated with a median survival of less than one year.

Diagnosis of Diffuse Large B-Cell Lymphoma. This large volume of malignant cells with abundant cytoplasm, nucleus round or oval type, prominent nucleoli, occasional mitotic diagnosis of diffuse large B-cell lymphoma (also known as immune lymphoma in conventional external inspection work, diffuse large B-cell lymphoma and metastatic carcinoma or need malignant melanoma, infectious mononucleosis syndrome, necrotizing lymphadenitis these types of diseases in the differential diagnosis.

\section{Common Treatments}

A Radiation Therapy. Radiation therapy was once a major radical treatment of early (I-II phase) malignant lymphoma. Radiotherapy in patients with stage I of the 5-year overall survival (OS) rate can reach $84 \%-90 \%$ in stage II patients up to $61 \%$. Since DLBCL is a systemic disease, at home and abroad, whether early or late, it is used to systemic chemotherapy treatment. For DLBCL with a large mass, after the full course of chemotherapy, MRD, conventional chemotherapy is not sensitive and specific part extranodal DLBCL patients with radiation therapy [3]. When a conventional domestic widespread treatment DLBCL, radiation tends to be over-applied, it has gradually improved. National Comprehensive Cancer Network (NCCN) guidelines state that, when combined treatment of DLBCL I-II, if not poor prognostic factors, may, after 3-4 cycles of immuno-chemotherapy, radiation therapy given to violations of the wild, you can achieve better clinical efficacy. Treatment of European countries are still emphasize adequate immuno-chemotherapy, if the patient achieved complete remission (CR) of the radiation is not necessary. At present, the concept of coexistence of the two 
treatments. We believe that currently used with chemotherapy treatment strategies, more in line with the biological behavior of DLBCL.

Conventional Chemotherapy. Since the 1970s, as the technology matures chemotherapy to CHOP regimen (cyclophosphamide, doxorubicin, vincristine, prednisone), represented by classical gradually replace radiotherapy and chemotherapy, or become highly malignant invasion standard treatment of NHL. From the early chemotherapy CVP (cyclophosphamide, vincristine, prednisone) program, the development of CSVP (cyclophosphamide, streptavidin melanin, vincristine, prednisone) program and is now used CHOP Program. Some patients will receive chemotherapy to cure. Late highly malignant DLBCL patients with conventional CHOP regimen can achieve good results, long-term survival of up to $40 \%-60 \%$. Late DLBCL treatment with standard chemotherapy with CHOP. CHOP-21 is usually 6-8 courses. In numerous studies abroad in CHOP program can greatly prolong survival in patients with advanced 5-year DFS rate of 40\%-55\%, 5-year OS rate of 40\%-65\%. Our clinicians also CHOP in a clinical study conducted numerous fl or retrospectively, with encouraging results. To further enhance the effect of chemotherapy [4]. There are many foreign clinical studies have shown that changing the dose intensity of chemotherapy, may improve the long-term effects, such as CHOP-14 or CHOP plus etoposide (CHOEP) and EPOCH and other programs can indeed improve some patients long-term effect in the country have been reported. In recent years, the clinical standard treatment has been generally accepted and applied, while universally accepted drug regimen containing anthracycline chemotherapy is the preferred DLBCL. For our patients cannot use immunity chemotherapy, more intense chemotherapy regimens may be effective, for example ACVBP, CHOEP and EPOCH programs for the elderly or infirm special patient populations, and also more and more use of low-dose anthracycline, liposomal doxorubicin chemotherapy, etc. effectively avoid unnecessary adverse reactions, to further improve the therapeutic effect.

Immuno-Chemotherapy. The last 10 years, with a monoclonal antibody (mAb) drugs such as anti-CD20 rituximab widely used chemotherapy ten rituximab (R-CHOP) programs have become standard immune chemotherapy for DLBCL.

France Bed GELA98.5 clinical studies first prove that rituximab may improve the outcome of patients with DLBCL conventional CHOP regimen in elderly (> 60 years), a 10-year randomized study (LNH-98.5) results further established the immuno-chemotherapy the clinical value, R-CHOP CHOP group and the group 10 years of progression-free survival (PFS) rates were $36.5 \%$ and $20.0 \%$ ( $\mathrm{P}<0.01)$, the median survival time

Of 8.4 years and 3.5 years, 10-year OS rates were $43.5 \%$ and $26.7 \%$ ( $\mathrm{P}<0.01$ ), respectively. Another American for randomized controlled study of elderly patients E4494 / C9793 also obtained similar results. R-CHOP, CHOP induction chemotherapy nine-year failure-free survival (FFS) rates were $35 \%$ and $25 \%$

$(\mathrm{P}=0.008)$, but there were no significant differences in adverse events. Young patients with low-risk DLBCL addition a multinational multicenter, randomized, controlled study (MInT) of 824 cases less than 60 years of using the R-CHOP and CHOP regimen group showed that R-CHOP and CHOP group 6-year event-free survival (EFS) rates were $74 \%$ and $56 \%(\mathrm{P}<0.01$ ), respectively, 6 -year OS rates were $90 \%$ and $80 \%$, respectively $(\mathrm{P}=001)$.

The Recurrence Salvage Therapy. At least 30\% -40\% of patients early after treatment remission will relapse after initial treatment for years. Although relapse after treatment worse than the first-line, if you use the proper rescue program for a further sequential autologous stem cell transplantation (ASCT) and other intensive treatment, some patients may still be cured, so efficient and low toxicity save vital programs. Rituximab is not only effective for newly diagnosed patients, and some patients relapse after remission can still improve the efficacy of rescue packages, especially after first-line chemotherapy have a longer disease-free survival time were. Now there is no standard salvage chemotherapy programs, including such as GDP, ICE and other programs is relatively good effect of gemcitabine (Gemcitabine) or platinum-based drugs. Try also abroad before stem cell collection for patients with rituximab treatment, in order to achieve the purpose of purifying the body. If the patient 
to remedy sensitive to chemotherapy, high-dose chemotherapy and ASCT could generally $45 \%$ to $50 \%$ of patients with long-term disease-free survival, similar to the results reported foreign phase.

\section{Chinese Medicine Treatment}

Currently Chinese medicine industry in general that "false, sputum, fatigue," is the basic cause of malignant lymphoma. Virtual ferrite due to inadequate or improper diet, or impassioned internal injuries, leading organs, meridians dysfunction, abnormal air-lift access, the phlegm, fatigue endogenous blood, sputum fatigue cement, not missed, blocked meridians and the cost disease. In addition, within the community physicians generally believe that the toxin is one of the causes of malignant lymphoma [5].

For malignant lymphoma pathogenesis, understanding modern doctors are basically the same, considered within the virtual, dysfunctional organs, leading to the body air-lift access disorders, within poor blood, sputum, fatigue evil life, which ultimately caused the sputum, addiction, drugs, cement, blocking the meridians, but missed Nanxiao cost disease.

In summary, modern doctors of TCM etiology and pathogenesis of malignant lymphoma awareness, according to more malignant lymphoma tumor visible surface sputum, Executive fruit Soin. Generated from the sputum of etiology, pathogenesis, and "riddled Jie sputum mischief" sputum Pathogenic characteristics of malignant lymphoma etiology, pathogenesis were discussed. Sputum, the spleen and liver and lung are closely related; to sputum, with the gas lift, can block the meridians, qi, blood caused fatigue; sputum changed with exogenous cult poison, toxin accumulation inside, fatigue and other blood knot and the disease. Further, each toxin awareness is not uniform, most of the system are made toxin, or cancer drugs, phlegm, made in the internal organs of the drug, this point of view, the nature of the toxin is not clear; small part He pointed out that wind-heat cult poison, toxic heat, toxic heat [6].

Aidi Injection. Aidi injection main component of plaques Ao, ginseng, Astragalus, Acanthopanax, used in a variety of clinical cancer treatment. Clinical studies have shown that a number of Aidi injection combined with chemotherapy in the treatment of malignant lymphoma attenuated synergistic effect of increasing the quality of life for patients; clinical reports alone Aidi injection in the treatment of lymphoma have a significant effect.

Compound Sophora Injection. Compound Sophora Injection The main components flavescens, clay answer, there is heat and dampness, cooling blood detoxification, Sanjie analgesic efficacy, clinical and more used in the adjuvant treatment of cancer pain with clinical findings in combination with chemotherapy, can improve efficacy, and reduce toxic side effects.

Cinobufacini Injection. Cinobufotalin is dried toad skin extract, detoxification, swelling, analgesic effect, and more used in the clinical treatment of advanced tumors, also used in the treatment of malignant lymphoma, and Western medicine combined with chemotherapy treatment discharge, a significant effect.

KLT. KLT main ingredient is provoked seedlings kernel oil, Clinical studies have shown that KLT combined with Western medicine radiotherapy and chemotherapy treatment of malignant lymphoma attenuated synergistic effect, reduce the relapse rate, and studies have shown that inhibition of lymphocyte .

Kang'ai Injection. Kang'ai injection mainly composed of Astragalus, ginseng, oxymatrine, clinical studies for the treatment of malignant lymphoma which may relieve side effects of chemotherapy, improving the quality of life.

\section{References}

[1] Deng Jiadong. Hematology [M] Shanghai Science and Technology Press 2001: 1061-1062.

[2] Zhang Zhinan, Shen Ti. Blood disease diagnosis and treatment standards [M], Beijing: Science Press, 2007. 
[3] Wu hongju, Zhang Qingyuan, Chen Defa. Comparative clinical study of rituximab combined with $\mathrm{CHOP}$ the $\mathrm{CHOP}$ in the treatment of newly diagnosed diffuse large B-cell lymphoma. Cancer, 2005, 24: 1498-1502.

[4] Xiazhong Jun, Wang Fenghua. Monoclonal antibody rituximab combined with CHOP treatment-naïve patients with diffuse large B-cell lymphoma of efficacy [J] Chinese Journal of Hematology. 2006(4), 4:27:

[5] Zhang Yacheng. Lymphoma Treatment Approaches on TCM [J]. Zhejiang Traditional Chinese Medicine, 201045 (4): 256 - 257

[6] Xiao Zhiwei. Professor Lin Lizhu experience in the treatment of malignant lymphoma [J] .2010 Hunan Traditional Chinese Medicine, 26 (3): 46--47 